\title{
Relatively-Integrated Ship Navigation by H¥ Fusion Filters
}

\author{
Yanping Yang, Yellow River Conservancy Technical Institute, China \\ Ruiguang Li, Henan University, China
}

\begin{abstract}
For the system with unknown statistical property noises, the property that the energies of the system noise and the observation noise are limited is utilized in this paper. On this basis, two novel fusion algorithms are proposed for ship-integrated navigation with the relative navigation information broadcasted by the automatic identification systems (AISs) in the adjacent ships. Firstly, an H¥ fusion filtering algorithm is given to deal with the navigation observation messages under the centralized fusion framework. The integrated navigation method based on this algorithm cannot deal with the asynchronous navigation messages in real time. Therefore, a sequential H¥ fusion-filtering algorithm is also given to sequentially deal with the asynchronous navigation messages. Finally, a computer simulation is employed to illustrate the validity and feasibility of the sequential method.
\end{abstract}

\section{KEYWORDS}

Asynchronous Navigation Messages, Fusion Filter, Integrated Navigation

\section{INTRODUCTION}

The integrated navigation systems are utilized in the largest number of modern ships (Yang,2009; Qiu, 2013; Xiong, 2001). The integrated navigation systems fuse the observations sampled by multiple navigation devices, such as, Global Position System (GPS), Inertial Navigation System (INS) and Celestial Navigation System (CNS), to obtain the more accurate and reliable navigational state information of the target ship, compared with the navigation system with single device (Wu, 2009; Li, 2014; Liu, 2011; Guo, 2015).

With the development of communication technology and computer science, the Automatic Identification System (AIS) has been growing popularity in ports and ships. The stars, ship-based radars, shore-based radars, AIS stations and maritime aircrafts make up a self-organizing wireless monitoring network to obtained various navigation information for ships. The ship equipped AIS broadcasts its ship identification, speed, course, location, and such other navigation state information to the shore-based AIS stations and other ships equipped AIS. It makes that the ship equipped AIS can obtain not only the observations sampled by its own navigation devices, but also the navigation state information about other ships in certain sea area, which may serve as the relative observation of the target ship. The adjacent ships in certain sea area around the target ship, in some sense, can be taken as the "relative navigation devices", which are the new navigation information source for 
the navigation systems. From the point of view of information processing, integrated utilizing the navigation information sampled by the inherent navigation devices in the target ship and the relative navigation information broadcasted by "relative navigation devices" is expected to obtain a more accurate and reliable navigational state information. It is called relative integrated navigation ( $\mathrm{Li}$, 2011). The work of (Ge, 2008) presented a ship relative integrated navigation algorithm to deal with the synchronous navigation observations obtained by the inherent navigation devices and relative navigation devices. The single difference of GPS pseudo-range was taken in account for the relative navigation problem (Wang, 2012). For the observation asynchronously sampled by multiple navigation devices or broadcasted by "relative navigation devices", a novel asynchronous integrated navigation method was also proposed (Ge, 2008). The delay phenomenon of transmitting the (relative) navigation information are considered (Liu, 2014), and a relative integrated navigation approach for the kinds of the scenario with delay navigation information was developed from the out-of-sequence-measurement fusion method. It is noted that most of the traditional integrated navigation methods (Yang, 2009; Qiu, 2013; Xiong, 2001; Wu, 2009; Li, 2014; Liu, 2011; Guo, 2015) and above relative integrated navigation methods (Ge, 2008; Wang, 2012; Liu, 2014) were all given on the basis of the assumption that the system noises and observation noises satisfy the Gaussian distribution with known statistic characteristics. However, the statistic characteristics of system noises and observation noises are usually difficult to accurately acquire in practical, because that the ships sailing on the sea are often disturbed by the wind, storm, surge, current and other sea condition or meteorological factors, which lead to complex yaw phenomenon and dynamic positioning location change.

Aiming at this problem, the property that the energies of the system noise and the observation noise are limited in finite horizon are utilized to describe the system. On this basis, two traditional integrated navigation methods are given (Liu, 2010; Zhao, 2002) to fuse the observations sampled by INS and GPS. These two methods, nevertheless, are both difficult to develop for the integrated navigation with observations sampled multiple general navigation devices, especially for the integrated navigation scenarios with relative navigation information. Therefore, it is the motivation of our research to design a novel relative integrated navigation method for general multiple navigation information sampled by the inherent navigation devices in the target ship and the relative navigation information broadcasted by "relative navigation devices".

In this paper, in the fusion framework of centralized fusion (Lin, 2003; Li, 2008; Xing, 2016) and sequential fusion (Feng, 2013; Bai, 2012), two H¥ fusion filter-based relative integrated navigation algorithms are proposed on the basis of the property that the energies of the system noise and the observation noise are limited in finite horizon are utilized. Firstly, an augmented observation vector is constructed including the navigation information sampled by the inherent navigation devices in the target ship and the relative navigation information broadcasted by adjacent ships in certain sea area around the target ship. On this basis, a fusion filtering performance index is defined and a centralized H¥ fusion filtering algorithm is presented with the augmented observation vector to satisfy the defined fusion filtering performance index. Due to the operating principles of different navigation devices, the operating frequencies of them usually differ. This results that the navigation observations are asynchronously sampled by different navigation devices. Therefore, the presented centralized H¥ fusion filtering algorithm lost the real time performance. In this text, the fusion H¥ fusion filtering algorithm are designed in a novel sequential structure. The defined fusion filtering performance index is re-described as a group of quadratic inequalities. On this basis, a sequential fusion filtering approach is proposed by sequentially solving the group of quadratic inequalities. The final computer simulation illustrated the feasibility and effectiveness of the proposed sequential fusion filtering method.

The rest of this paper is organized as follows. Section 1 describes the dynamic models of the target ship and the adjacent ships, which are further rewritten as the relative navigation devices. In Section 2, two ship relative integrated navigation methods are proposed based on the finite horizon H¥ filter, under the centralized fusion framework and the sequential fusion framework, sequentially. 
A simple example of application is presented to illustrate the validity and feasibility of the sequential method in Section 3 and Section 4 summarizes the main contribution of this paper.

\section{DYNAMIC MODELS}

Consider the dynamic models of the target ship and adjacent ships in certain sea area can be modeled as

$$
\mathbf{x}_{i}(k+1)=\mathbf{F}_{i}(k+1, k) \mathbf{x}_{i}(k)+\mathbf{w}_{i}(k+1, k), i=1,2, \cdots, N
$$

Without loss of generality, label the target ship as 1 , and label the adjacent ships which broadcast relative navigation information as $2, \cdots, N$. In $(1), \mathbf{x}_{i}(k)=\left[x_{i}(k), y_{i}(k), v_{i}(k), \varphi_{i}(k)\right]^{T}$ is the ship' Navigational state vector, where $x_{i}(k), y_{i}(k), v_{i}(k), \varphi_{i}(k)$ are respectively the position of east, the position of north, the speed over ground and the course over ground of the ship $i, i=1,2, \cdots, N$. (in which, the speed of the Ocean-going vessel usually not exceeding $30 \mathrm{~nm} / \mathrm{h}) . \mathbf{F}_{i}(k+1, k)$ is the state transfer matrix of ship $i$ from $k$ to $k+1$, and $\mathbf{w}_{i}(k+1, k)$ is the corresponding accumulative system noise, the statistical property of which is unknown. The property that the energies of the system noise is limited in finite horizon is utilized in this paper, namely, $\sum_{k=0}^{T}\left(\mathbf{w}_{i}^{T}(k+1, k) \mathbf{w}_{i}(k+1, k)\right)<\infty$.

Two kinds of navigation information can be obtained by the target ship: the one sampled by the inherent navigation devices of the target ship, and the one broadcasted by the AIS in the adjacent ships in certain sea area around the target ship. These observations can be preprocessed and described as follows

$$
\mathbf{y}_{l}(k)=\mathbf{H}_{l}(k) \mathbf{x}_{1}(k)+\mathbf{v}_{l}(k) l=1,2, \cdots, M(k)
$$

where $M(k)$ is the number of the inherent navigation devices and relative navigation devices which provide the target ship with (relative) navigation information. $\mathbf{y}_{l}(k)$ is the (relative) navigation observation of the $l$ th device, $\mathbf{H}_{l}(k)$ is the corresponding observation matrix, and $\mathbf{v}_{l}(k)$ is the corresponding observation noise with unknown statistical property, namely, $\sum_{k=0}^{T}\left(\mathbf{v}_{i}^{T}(k) \mathbf{v}_{i}(k)\right)<\infty$. Denote the signal to be estimated in the navigation of target ship as

$$
\mathbf{z}(k)=\mathbf{L}(k) \mathbf{x}_{1}(k)
$$

Remark 1: If $\mathbf{L}(k)=I, \mathbf{z}(k)=\mathbf{x}_{1}(k)$. It means that all the navigation state vector is focused. The other forms of the signal to be estimated can also be given for other purposes. For instance, the position of target ship is payed more attention, then $\mathbf{L}(k)=\left[\begin{array}{ll}\mathbf{I}_{2 \times 2} & \mathbf{0}_{2 \times 2}\end{array}\right]$.

\section{THE RELATIVE INTEGRATED NAVIGATION METHODS}

For the system with the assumption that system noise and observation noise are both satisfy Gaussian distribution with known means and variances, multiple integrated navigation methods have been developed on the basis of Kalman filter in the scenario with synchronous observations, asynchronous observations, and delay observations. However, the statistical properties of the system noise and 
observation noise are usually difficult to accurately acquire in short time, in practical. Therefore, the property that the energies of the system noise and the observation noise are limited is utilized in this paper. It means that $\sum_{k=0}^{T}\left(\mathbf{w}_{i}^{T}(k+1, k) \mathbf{w}_{i}(k+1, k)\right)<\infty$, and $\sum_{k=0}^{T}\left(\mathbf{v}_{l}^{T}(k) \mathbf{v}_{l}(k)\right)<\infty$.

Denote the estimate of the signal to be estimated $\mathbf{z}(k)$ as $\hat{\mathbf{z}}(k \mid k)$, and the estimation error is $e_{z}(k \mid k):=\mathbf{z}(k)-\hat{\mathbf{z}}(k \mid k)$. The H¥ fusion filter to be designed for the relative integrated navigation method should satisfy the following performance index:

$$
\sup _{\mathbf{w}, \mathbf{v}} \frac{\sum_{i=1}^{k} \mathbf{e}_{z}^{T}(i) \mathbf{e}_{z}(i)}{\sum_{i=1}^{k} \sum_{l=1}^{M(k)} \mathbf{v}_{l}^{T}(i) \mathbf{v}_{l}(i)+\sum_{i=1}^{k} \mathbf{w}^{T}(i, i-1) \mathbf{w}(i, i-1)+\left(\mathbf{x}(0)-\hat{\mathbf{x}}_{0}\right)^{T} \mathbf{P}_{0}^{-1}\left(\mathbf{x}(0)-\hat{\mathbf{x}}_{0}\right)}<\gamma^{2}
$$

When all observations $\left\{\mathbf{y}_{l}(k) \mid l=1,2, \cdots, M(k)\right\}$ sampled at $k$ are all received by the signal processing center, some auxiliary augmented matrices are defined as follows:

$$
\mathbf{Y}(k)=\left[\begin{array}{c}
\mathbf{y}_{1,}(k) \\
\vdots \\
\mathbf{y}_{M(k)}(k)
\end{array}\right], \widehat{H}(k)=\left[\begin{array}{c}
\mathbf{H}_{1}(k) \\
\vdots \\
\mathbf{H}_{M(k)}(k)
\end{array}\right], \mathbf{V}(k)=\left[\begin{array}{c}
\mathbf{v}_{1}(k) \\
\vdots \\
\mathbf{v}_{M(k)}(k)
\end{array}\right]
$$

Then the augmented observation equation can be described as

$$
\mathbf{Y}(k)=\widehat{\mathbf{H}}(k) \mathbf{x}_{1}(k)+\mathbf{V}(k)
$$

and the performance index defined in (4) can be furtherly rewritten with the augmented matrices given in (5) as:

$$
\sup _{\mathbf{w}, \mathbf{V}} \frac{\sum_{i=1}^{k} \mathbf{e}_{z}^{T}(i) \mathbf{e}_{z}(i)}{\sum_{i=1}^{k} \mathbf{V}^{T}(i) \mathbf{V}(i)+\sum_{i=1}^{k} \mathbf{w}^{T}(i, i-1) \mathbf{w}(i, i-1)+\left(\mathbf{x}(0)-\hat{\mathbf{x}}_{0}\right)^{T} \mathbf{P}_{0}^{-1}\left(\mathbf{x}(0)-\hat{\mathbf{x}}_{0}\right)}<\gamma^{2}
$$

Considering the noise property $\sum_{k=0}^{T}\left(\mathbf{w}_{i}^{T}(k+1, k) \mathbf{w}_{i}(k+1, k)\right)<\infty$, and $\sum_{k=0}^{T}\left(\mathbf{V}_{l}^{T}(k) \mathbf{V}_{l}(k)\right)<\infty$, the following centralized H¥ fusion filtering algorithm is presented for the integrated navigation with $\mathbf{Y}(k)$.The estimate of the signal to be estimated $\mathbf{z}(k)$ is given by

$$
\hat{\mathbf{z}}(k \mid k)=\mathbf{L}(k) \hat{\mathbf{x}}_{1}(k \mid k)
$$

where 


$$
\begin{aligned}
& \hat{\mathbf{x}}_{1}(k \mid k)=\mathbf{F}_{1}(k, k-1) \hat{\mathbf{x}}_{1}(k-1 \mid k-1)+\mathbf{K}(k)\left[\mathbf{Y}(k)-\hat{\mathbf{H}}(k) \mathbf{F}_{1}(k, k-1) \hat{\mathbf{x}}_{1}(k-1 \mid k-1)\right] \\
& \mathbf{K}(k)=\mathbf{P}(k) \widehat{\mathbf{H}}^{T}(k)\left[\widehat{\mathbf{H}}(k) \mathbf{P}(k) \widehat{\mathbf{H}}^{T}(k)+\mathbf{I}\right]^{-1} \\
& \mathbf{P}(k)=\mathbf{F}_{1}(k, k-1) \mathbf{P}(k-1) \mathbf{F}_{1}^{T}(k, k-1)+\mathbf{I} \\
& -\mathbf{F}_{1}(k, k-1) \mathbf{P}(k-1)\left[\widehat{\mathbf{H}}^{T}(k-1) \quad \mathbf{L}^{T}(k-1)\right] \mathbf{R}_{e}^{-1}(k-1)\left[\begin{array}{c}
\widehat{\mathbf{H}}(k-1) \\
\mathbf{L}(k-1)
\end{array}\right] \mathbf{P}(k-1) \mathbf{F}_{1}^{T}(k, k-1) \\
& \mathbf{R}_{e}(k-1)=\left[\begin{array}{cc}
\mathbf{I} & \mathbf{0} \\
\mathbf{0} & -\gamma^{2} \mathbf{I}
\end{array}\right]+\left[\begin{array}{c}
\widehat{\mathbf{H}}(k-1) \\
\mathbf{L}(k-1)
\end{array}\right] \mathbf{P}(k-1)\left[\widehat{\mathbf{H}}^{T}(k-1) \quad \mathbf{L}^{T}(k-1)\right]
\end{aligned}
$$

The existing condition of the centralized H¥ fusion filtering algorithm above is

$$
\mathbf{P}^{-1}(k)+\left[\begin{array}{c}
\widehat{\mathbf{H}}(k-1) \\
\mathbf{L}(k-1)
\end{array}\right]\left[\begin{array}{cc}
\mathbf{I} & \mathbf{0} \\
\mathbf{0} & -\gamma^{-2} \mathbf{I}
\end{array}\right]\left[\begin{array}{ll}
\hat{\mathbf{H}}^{T}(k-1) & \mathbf{L}^{T}(k-1)
\end{array}\right]>0
$$

Remark 2: The integrated navigation based on above H¥ fusion filtering method deals with not only the navigation information sampled by the inherent devices (GPS, INS, and so on) in the target ship, but also the relative navigation information broadcasted from the AIS in the adjacent ships, to improve the navigation accuracy of the target ship. It is noted that the H¥ fusion filtering method above is designed in the centralized fusion frame, which deal with all the (relative) navigation information sampled at $k$ in one time. It is implied that the signal processing center cannot deal with the (relative) navigation information until all of them received by the signal processing center. Nevertheless, due to the fact that the operating principles of each device is usually different from others, the operating frequencies of them often differ. It means that the integrated navigation based on above H¥ fusion filtering method cannot always provide the real time navigation state estimate, especially for the scenarios with asynchronously sampling of navigation devices or delay phenomenon of communication networks.

To address the issue mentioned in Remark 2, furtherly, the H¥ fusion filter that constraints of the performance index (4) is re-designed in the sequential fusion frame, which deal with the (relative) navigation observations in their received order by the information processing center. The sequential H¥ fusion filter can not only deal with the synchronous observations, but also is effective for the observations are sampled asynchronously or transmitted with short-time delay phenomenon.

In order to design an H¥ fusion filter in the sequential fusion frame to satisfy the performance index (4), the performance index is re-described as the following quadratic inequality 


$$
\begin{aligned}
\mathbf{J}(k)= & \sum_{i=1}^{k} \mathbf{w}^{T}(i, i-1) \mathbf{w}(i, i-1)+\left(\mathbf{x}(0)-\hat{\mathbf{x}}_{0}\right)^{T} \mathbf{P}_{0}^{-1}\left(\mathbf{x}(0)-\hat{\mathbf{x}}_{0}\right) \\
& \quad+\sum_{i=1}^{k}\left(\sum_{l=1}^{M(k)} \mathbf{v}_{l}^{T}(i) \mathbf{v}_{l}(i)-\gamma^{-2} \mathbf{e}_{z}^{T}(i) \mathbf{e}_{z}(i)\right) \\
= & \mathbf{J}(k-1)+\mathbf{w}^{T}(k, k-1) \mathbf{w}(k, k-1)+\sum_{l=1}^{M(k)} \mathbf{v}_{l}^{T}(k) \mathbf{v}_{l}(k)-\gamma^{-2} \mathbf{e}_{z}^{T}(k) \mathbf{e}_{z}(k)>0
\end{aligned}
$$

The performance index above is equivalent to the following quadratic inequality group

$$
\left\{\begin{array}{l}
\mathbf{J}_{1}(k)=\mathbf{J}(k-1)+\mathbf{w}^{T}(k, k-1) \mathbf{w}(k, k-1)+\mathbf{v}_{1}^{T}(k) \mathbf{v}_{1}(k)>0 \\
\mathbf{J}_{i}(k)=\mathbf{J}_{i-1}(k)+\mathbf{v}_{i}^{T}(k) \mathbf{v}_{i}(k)>0, \quad i=2, \cdots, N-1 \\
\mathbf{J}_{N}(k)=\mathbf{J}_{N-1}(k)+\mathbf{v}_{N}^{T}(k) \mathbf{v}_{N}(k)-\gamma^{-2} \mathbf{e}_{z}^{T}(k) \mathbf{e}_{z}(k)>0
\end{array}\right.
$$

Solve above quadratic inequalities, sequentially. The approach of sequential H¥ fusion filtering is given as follows:

$$
\left.\begin{array}{l}
\hat{\mathbf{x}}_{1,1}(k \mid k)=\mathbf{F}(k, k-1) \hat{\mathbf{x}}_{1}(k-1 \mid k-1)+\mathbf{K}_{1}(k)\left[\mathbf{y}_{1}(k)-\mathbf{H}_{1}(k) \mathbf{F}_{1}(k, k-1) \hat{\mathbf{x}}_{1}(k-1 \mid k-1)\right] \\
\hat{\mathbf{z}}_{1}(k \mid k)=\mathbf{L}(k) \hat{\mathbf{x}}_{1,1}(k \mid k) \\
\mathbf{K}_{1}(k)=\mathbf{P}_{1}(k) \mathbf{H}_{1}^{T}(k)\left(\mathbf{H}_{1}(k) \mathbf{P}_{1}(k) \mathbf{H}_{1}^{T}(k)+\mathbf{I}\right)^{-1} \\
\mathbf{P}_{2}(k)=\mathbf{P}_{1}(k)-\mathbf{P}_{1}(k) \mathbf{H}_{1}^{T}(k)\left(\mathbf{H}_{1}(k) \mathbf{P}_{1}(k) \mathbf{H}_{1}^{T}(k)+\mathbf{I}\right)^{-1} \mathbf{H}_{1}(k) \mathbf{P}_{1}(k) \\
\hat{\mathbf{x}}_{1,2}(k \mid k)=\hat{\mathbf{x}}_{1,1}(k \mid k)+\mathbf{K}_{2}(k)\left[\mathbf{y}_{2}(k)-\mathbf{H}_{2}(k) \hat{\mathbf{x}}_{1,1}(k \mid k)\right] \\
\hat{\mathbf{z}}_{2}(k \mid k)=\mathbf{L}(k) \hat{\mathbf{x}}_{1,2}(k \mid k) \\
\mathbf{K}_{2}(k)=\mathbf{P}_{2}(k) \mathbf{H}_{2}{ }^{T}(k)\left(\mathbf{H}_{2}(k) \mathbf{P}_{2}(k) \mathbf{H}_{2}^{T}(k)+\mathbf{I}\right)^{-1} \\
\mathbf{P}_{3}(k)=\mathbf{P}_{2}(k)-\mathbf{P}_{2}(k) \mathbf{H}_{2}^{T}(k)\left(\mathbf{H}_{2}(k) \mathbf{P}_{2}(k) \mathbf{H}_{2}^{T}(k)+\mathbf{I}\right)^{-1} \mathbf{H}_{2}(k) \mathbf{P}_{2}(k)
\end{array}\right\}
$$

$$
\left.\begin{array}{l}
\left.\hat{\mathbf{x}}_{1, M(k)-1}(k \mid k)=\hat{\mathbf{x}}_{1, M(k)-2}(k \mid k)+\mathbf{K}_{M(k)-1}(k)\left[\mathbf{y}_{M(k)-1}(k)-\mathbf{H}_{M(k)-1}(k) \hat{\mathbf{x}}_{1, M(k)-2}(k \mid k)\right]\right] \\
\hat{\mathbf{z}}_{M(k)-1}(k \mid k)=\mathbf{L}(k) \hat{\mathbf{x}}_{1, M(k)-1}(k \mid k) \\
\mathbf{K}_{M(k)-1}(k)=\mathbf{P}_{M(k)-1}(k) \mathbf{H}_{M(k)-1}^{T}(k)\left(\mathbf{H}_{M(k)-1}(k) \mathbf{P}_{M(k)-1}(k) \mathbf{H}_{M(k)-1}^{T}(k)+\mathbf{I}\right)^{-1} \\
\mathbf{P}_{M(k)}(k)=\mathbf{P}_{M(k)-1}(k)-\mathbf{P}_{M(k)-1}(k) \mathbf{H}_{M(k)-1}^{T}(k) \\
\quad \times\left(\mathbf{H}_{M(k)-1}(k) \mathbf{P}_{M(k)-1}(k) \mathbf{H}_{M(k)-1}^{T}(k)+\mathbf{I}\right)^{-1} \mathbf{H}_{M(k)-1}(k) \mathbf{P}_{M(k)-1}(k)
\end{array}\right\}
$$




$$
\begin{aligned}
& \hat{\mathbf{x}}_{1, M(k)}(k \mid k)=\hat{\mathbf{x}}_{1, M(k)-1}(k \mid k)+\mathbf{K}_{M(k)}(k)\left(\mathbf{y}_{M(k)}(k)-\mathbf{H}_{M(k)}(k) \hat{\mathbf{x}}_{1, M(k)-1}(k \mid k)\right) \\
& \hat{\mathbf{z}}_{M(k)}(k \mid k)=\mathbf{L}(k) \hat{\mathbf{x}}_{1, M(k)}(k \mid k) \\
& \mathbf{K}_{M(k)}(k)=\mathbf{P}_{M(k)}(k) \mathbf{H}_{M(k)}^{T}(k)\left(\mathbf{H}_{M(k)}(k) \mathbf{P}_{M(k)}(k) \mathbf{H}_{M(k)}^{T}(k)+\mathbf{I}\right)^{-1} \\
& \mathbf{P}_{1}(k+1)=\mathbf{F}_{1}(k+1, k) \mathbf{P}_{M(k)}(k) \mathbf{F}_{1}^{T}(k+1, k)+\mathbf{I} \\
& -\mathbf{F}_{1}(k+1, k) \mathbf{P}_{M(k)}(k)\left[\begin{array}{ll}
\mathbf{H}_{M(k)}^{T}(k) & \mathbf{L}^{T}(k)
\end{array}\right] \mathbf{R}_{e}^{-1}(k)\left[\begin{array}{c}
\mathbf{H}_{M(k)}(k) \\
\mathbf{L}(k)
\end{array}\right] \mathbf{P}_{M(k)}(k) \mathbf{F}_{1}^{T}(k+1, k) \\
& \mathbf{R}_{e}(k)=\left[\begin{array}{cc}
\mathbf{I} & \mathbf{0} \\
\mathbf{0} & -\gamma^{2} \mathbf{I}
\end{array}\right]+\left[\begin{array}{c}
\mathbf{H}_{M(k)}(k) \\
\mathbf{L}(k)
\end{array}\right] \mathbf{P}_{M(k)}(k)\left[\begin{array}{ll}
\mathbf{H}_{M(k)}^{T}(k) & \mathbf{L}^{T}(k)
\end{array}\right]
\end{aligned}
$$

The existing condition of above approach of sequential H¥ fusion filtering is given by

$$
-\gamma^{2} \mathbf{I}+\mathbf{L}(k)\left(\mathbf{P}_{M(k)}^{-1}(k)+\mathbf{H}_{M(k)}^{T}(k) \mathbf{H}_{M(k)}(k)\right)^{-1} \mathbf{L}^{T}(k)<0
$$

Remark 3: When the first (relative) observation about the navigation state at $k$ is received by the information processing center, the navigation state estimate at $k$ can be obtained by (15), without waiting for other observations. When other (relative) observation about the navigation state at $k$ is received again, the navigation state estimate at $k$ can be updated by (16)-(18). At the same time, the estimate of the signal payed more attention can also be obtained. The flow chart of the above algorithm of sequential fusion filtering is given as follows.

\section{NUMERICAL SIMULATION}

Consider a target ship navigates with constant course and constant speed, and there are two adjacent ships in certain sea area around the target ship, which provide their navigation information as the relative navigation observations. Assume that the two adjacent ships also navigate with constant course and constant speed. Therefore, the state transfer matrix of each ship's dynamic models can be described as

$$
\mathbf{F}_{i}(k+1, k)=\left[\begin{array}{cccc}
1 & 0 & \cos \varphi_{i} T_{i} & 0 \\
0 & 1 & \sin \varphi_{i} T_{i} & 0 \\
0 & 0 & 1 & 0 \\
0 & 0 & 0 & 1
\end{array}\right], i=1,2,3
$$

where $\varphi_{1}=60^{\circ}, \varphi_{2}=30^{\circ}, \varphi_{3}=30^{\circ}, T_{i}=12 s, i=1,2,3$.

Assume the target ship's speed over ground is $12 \mathrm{~nm} / \mathrm{h}$, the speeds over ground of the adjacent ship 1 and the adjacent ship 2 are $14 \mathrm{~nm} / \mathrm{h}$ and $16 \mathrm{~nm} / \mathrm{h}$, separately. The courses over ground of them are separately $\varphi_{1}=60^{\circ}, \varphi_{2}=30^{\circ}, \varphi_{3}=30^{\circ}$. There are two inherent navigation devices in each ship, and the sampling period is $12 \mathrm{~s}$. The observation matrix of each navigation device is unit matrix with appropriate dimension. The statistical properties of the observation noises and system noises are unknown. The start navigation states of three ships are given by 
Figure 1. The position curse of three ships

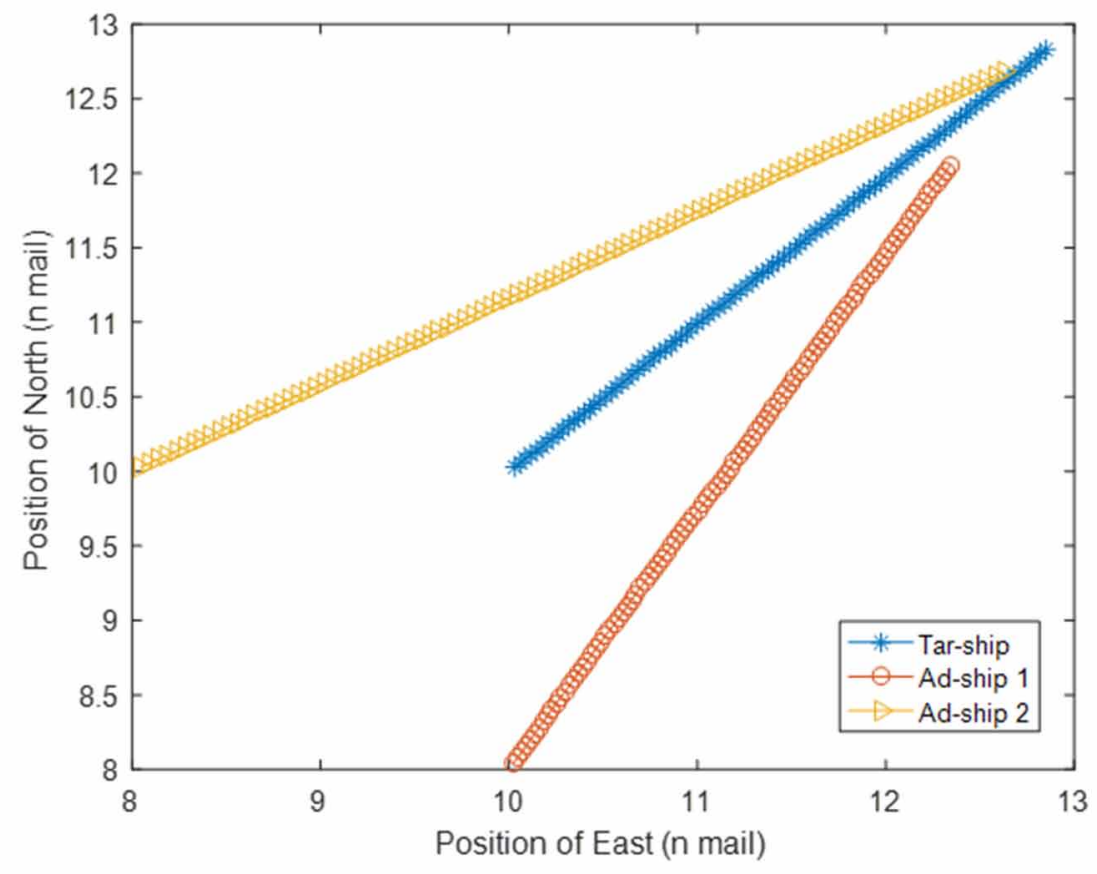

$\left\{\begin{array}{l}\mathbf{x}_{1}(0)=[10,5,10, \pi / 4.5]^{T} \\ \mathbf{P}_{1}(0)=\operatorname{diag}\{10,10,50,1\}\end{array} \quad\left\{\begin{array}{l}\mathbf{x}_{2}(0)=[15,6,12, \pi / 6]^{T} \\ \mathbf{P}_{2}(0)=\operatorname{diag}\{10,10,50,1\}\end{array}\left\{\begin{array}{l}\mathbf{x}_{3}(0)=[15,9,10, \pi / 3]^{T} \\ \mathbf{P}_{3}(0)=\operatorname{diag}\{10,10,50,1\}\end{array}\right.\right.\right.$

Take the target ship's position of east and position of north as the signal to be estimated, then $\mathbf{z}(k)=\mathbf{L}(k) \mathbf{x}(k)$, and $\mathbf{L}(k)=\left[\begin{array}{ll}\mathbf{I}_{2 \times 2} & \mathbf{0}_{2 \times 2}\end{array}\right]$. Set the performance index value is $\gamma=3$.

Due to the statistical properties of the measurement noises and the process noises are all unknown. The performance that the energy of the noises in short time period is finite is utilized, in this paper, to describe the noises, namely, $\sum_{k=0}^{T}\left(\mathbf{w}_{i}^{T}(k+1, k) \mathbf{w}_{i}(k+1, k)\right)<\infty, \sum_{k=0}^{T}\left(\mathbf{v}_{i}^{T}(k) \mathbf{v}_{i}(k)\right)<\infty, i=1,2,3$.

In this simulation, the relative integrated navigation approach based on the above algorithm of sequential fusion filtering in (15)-(18) is utilized. The simulation results are given as follows, in which the target ship is short for "Tar-ship", and the two adjacent ships are separately denoted as "Ad-ship 1" and "Ad-ship 2", and "AEEP" is Absolute Estimate Error of Position.

As shown in the simulation results above, the relative integrated navigation approach based on the algorithm of sequential fusion filtering (15)-(17) can effectively deal with the observations sampled by the inherent devices and the relative observations broadcasted by the adjacent ships, even the system noises and observation noises are with unknown statistical properties. 
Figure 2. The position estimate of the target ship

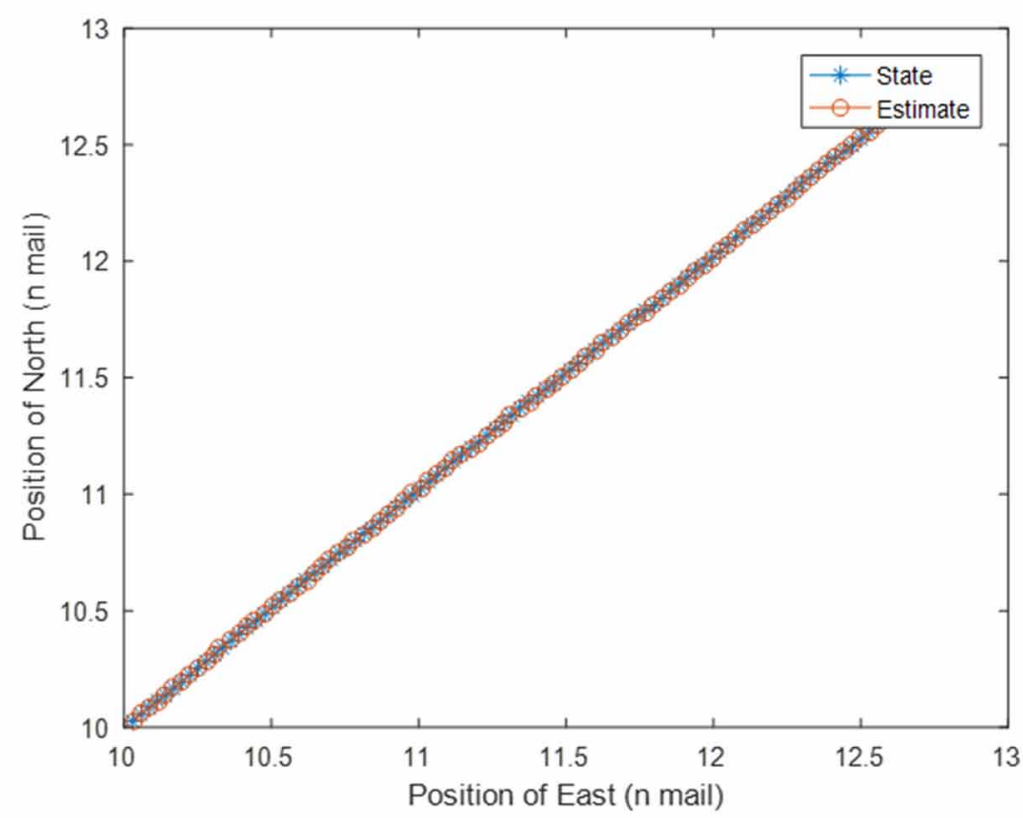

Figure 3. Absolute Estimate Error of east Position

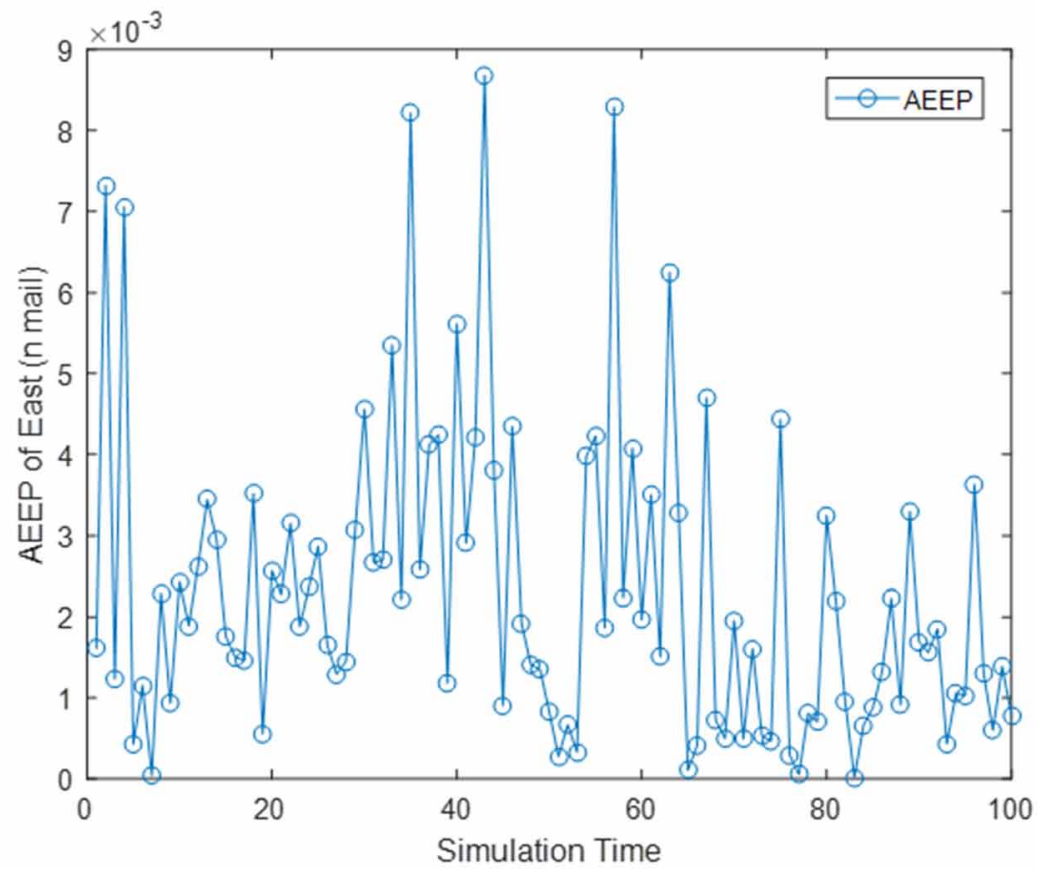




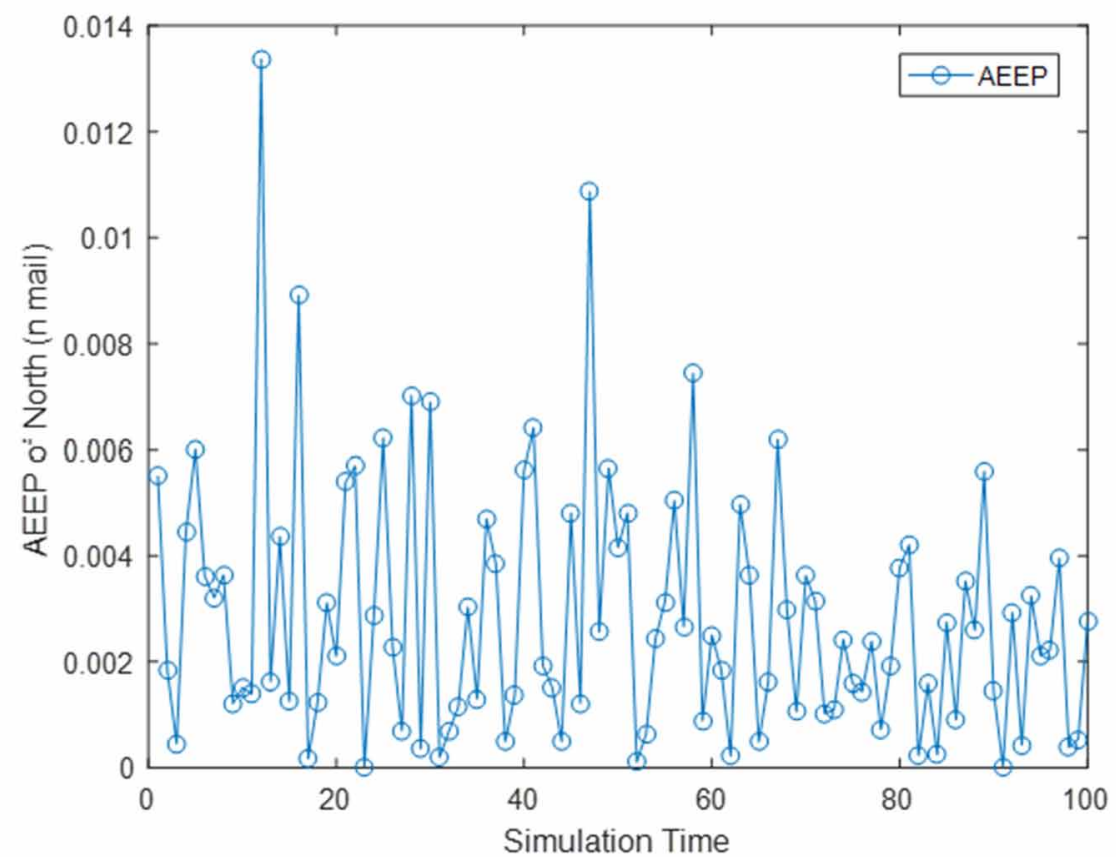

\section{CONCLUSION}

Most of the existing integrated navigation methods are developed from the famous Kalman filter, based on the assumption that the system noise and observation noises satisfy Gaussian distribution. However, the statistical properties of system noise and observation noises are difficult to accurately obtain in short time. In this paper, the relative integrated navigation is addressed without the assumption, the property that the energies of the noises are limited is utilized to design two approaches of H¥ fusion filtering. The method based on the given centralized H¥ fusion filtering deals with all the navigation observations synchronously received. For the asynchronous observations, the method on the basis of sequential H¥ fusion filtering ensures the real time property of relative integrated navigation. It 
should be noted that the performance index value is given as a constant. It is implied that the state estimates of the target ship is suboptimal. One of the further works is to study the optimal relative integrated navigation methods.

\section{REFERENCES}

Feng, X., Wen, C., \& Xu, L. (2013). Multi-sensor multi-OOSM distributed sequential fusion filtering. Proceedings of 2013 IEEE International Conference on Control and Automation, 772-776.

Ge, Q. (2008). Networked fusion with out-of-Sequence information and its application in ship integrated navigation. Shanghai Maritime University.

Hang, G., Guo, J., Min, Y., Hong, H., Jian, X., \& Tian, B. (2015). A weighted combination filter with nonholonomic constrains for integrated navigation systems. Advances in Space Research, 55(5), 1470-1476. doi:10.1016/j.asr.2014.12.005

Li, J., Li, H., Tang, G., \& Luo, Y. Z. (2011). Research on the strategy of angles-only relative navigation for autonomous rendezvous. Science China. Technological Sciences, 54(7), 1865-1872. doi:10.1007/s11431-011$4442-9$

Li, Q., Wang, H., Hua, L., Liu, X., \& Fei, L. (2008). Centralized H¥ fusion filter design in multi-sensor data fusion system. Proceedings of 2008 IEEE Conference on Robotics, Automation and Mechatronics, 603-607. doi:10.1109/RAMECH.2008.4681336

Lin, Y., Xue, A., \& Qian, J. (2003) Discrete-time H¥ fusion filter in data fusion system. Proceedings of 2003 IEEE Conference on Control Applications, 1420-1424.

Liu, M., \& Xiong, F. (2011). A fuzzy adaptive GPS/INS integrated navigation algorithm. Procedia Engineering, 15, 660-664. doi:10.1016/j.proeng.2011.08.123

Meng, B., \& Li, M. (2012). Application of an adaptive sequential Kalman filter to SINS/GPS navigation data fusion. Proceedings of 2012 World Congress on Intelligent Control and Automation, 4309-4314.

Ming, L., Feng, X., Shi, J., \& Qiu, A. (2014). Optimal positioning algorithm for integrated relative navigation of ships. Navigation of China, 37(4), 6-11.

Qiu, A., Shi, J., Feng, X., \& Guan, B. (2013). A navigational information fusion algorithm based on model library. Navigation of China, 36(3), 5-9.

Wang, X., Zhou, H., \& Zhang, S. (2012). Relative navigation between vessels based on GPS single difference. Journal of Chinese Inertial Technology, 20(4), 464-467.

Wei, , Liangjie, Haina, \& Yufeng. (2001). Method of federated Kalman filtering in INS/ESGM/Doppler integrated navigation system. Journal of Chinese Inertial Technology, 9(1), 1-11.

Wei, Z., Xin, Y., \& Lin, X. (2002). Research on GPS /INS integration based on H¥ filter. Journal of Astronautics, 23(3), 39-43.

Wu, J., \& Zhang, H. (2009). Data fusion algorithm design of GPS/IMU based on fuzzy adaptive federated Kalman filter. Proceedings of the 2nd International Congress on Image and Signal Processing, 1-4. doi:10.1109/ CISP.2009.5301061

Xing, Z., \& Xia, Y. (2016). Comparison of centralised scaled unscented Kalman filter and extended Kalman filter for multisensor data fusion architectures. IET Signal Processing, 10(4), 359-365. doi:10.1049/iet-spr.2015.0205

Yang, R., Yun'an, H., Fan, Y., \& Geng, B. (2009). Prospect and research of marine integrated navigation technology. Journal of Naval Aeronautical and Astronautical University, 24(1), 47-51.

Yong, L. (2014). Optimal multisensor integrated navigation through information space approach. Physical Communication, 13, 44-53. doi:10.1016/j.phycom.2013.11.011

Yu, L., Wang, H., \& Gao, S. (2010). Research on technology of tightly integrated navigation based on H¥ filtering the high dynamic circumstance. Fire Control \& Command Control, 35(11), 184-187. 
Yanping Yang received her BSc degree in Henan University and her MSc degree in East China Normal University. She is currently a lecturer in the Department of Basic, Yellow River Conservancy Technical Institute. Her current research interests include stochastic mathematics, information processing.

Ruiguang Li received his MSc degree in School of Computer and Information Engineering, Henan University. He currently works in the College English Department, Henan University. His research interests include stochastic information processing. 